\title{
5' Splice site selection in yeast: genetic alterations in base-pairing with U1 reveal additional requirements
}

\author{
Paul G. Siliciano and Christine Guthrie \\ Department of Biochemistry and Biophysics, University of California at San Francisco, \\ San Francisco, California 94143 USA
}

\begin{abstract}
Using a strategy of compensatory nucleotide changes between yeast $\mathrm{U} 1$ and a $5^{\prime}$ splice site, we have analyzed the contribution of base-pairing to the efficiency and fidelity of pre-mRNA splicing in vivo. Watson-Crick base-pairing interactions with $U 1$ can be demonstrated at intron positions 1 and 5 but not at position 4 . Moreover, restoration of the ability to pair with $\mathrm{U} 1$ is not sufficient to restore activity in the second step of splicing to intron position 1 mutants. Finally, in contrast to recent observations in mammalian systems, we find that the precise position of $5^{\prime}$ splice site cleavage is not determined solely by the base-pairing interaction with U1. Rather, the presence of a $G$ residue at position 5 is required for the correct localization of the nucleolytic event. Taken together, these results indicate that the demands for $5^{\prime}$ splice site selection and utilization are more complex than a simple maximization of Watson-Crick interactions with U1.
\end{abstract}

[Key Words: Splicing; 5'splice site; U1 snRNA]

Received June 22, 1988; revised version accepted August 18, 1988.

Understanding the specificity of splice site selection remains one of the most central problems in pre-mRNA splicing. Since the potential complementarity between the conserved $5^{\prime}$ end of the U1 snRNA and the consensus sequence spanning the $5^{\prime}$ splice site (CAG/GUPuAGU) was first noted (Lerner et al. 1980; Rogers and Wall 1980|, specific hypotheses have focused on the role of base-pairing between conserved intron signals and the snRNAs. Numerous biochemical experiments indicated that U1 is required for splicing (Kramer et al. 1984; Black et al. 1985; Krainer and Maniatis 1985), that it binds to the $5^{\prime}$ splice site (Mount et al. 1983), and that this binding depends on the 5 ' end of U1 (Chabot et al. 1985).

Zhuang and Weiner (1986) first demonstrated specific base-pairing interactions between $\mathrm{Ul}$ and the $5^{\prime}$ splice site by achieving in vivo suppression of $5^{\prime}$ splice site mutants via compensatory changes in Ul. Splicing of adenovirus E1A transcripts carrying a $G \rightarrow A$ transition at the fifth position of the intron (herein designated by the convention IVS-A5) was efficiently restored by expression of a mutant $U 1$ with a compensatory change at position $4(\mathrm{Ul}-4 \mathrm{U})$. By the same assay, however, suppression of mutants at intron position 3 was found to be very inefficient, at best (Zhuang and Weiner 1986). Other experiments (in which only the intron was changed) showed that mutation of the SV40 large T antigen $5^{\prime}$ splice site to improve its match to the consensus results in an increased usage of this site, relative to the small t 5 ' splice site, both in vivo and in vitro (Zhuang et al. 1987). In combination with other studies (see below), the view has emerged that the net strength of basepairing interactions is a crucial determinant of the effi- ciency of 5 ' splice site utilization, particularly when several potential sites are competing in cis.

Moreover, the results of a systematic analysis of mutations at intron positions 1 and 2 suggest that the precise site of $5^{\prime}$ cleavage is not determined by the highly conserved intron sequence /GU but most likely by the specific location of certain residues of $\mathrm{U} 1$ within the base-paired region (Aebi et al. 1987; Weber and Aebi 1988). A series of 5 ' splice site mutations was designed that shift the complementarity to Ul relative to the intron sequences. These workers found that the site of $5^{\prime}$ cleavage is not fixed in reference to the conserved intron sequences but shifts according to the complementarity to Ul. In fact, the specific site of $5^{\prime}$ cutting in the intron could be predicted as that bond opposing the $\mathrm{C} 8-\mathrm{C} 9$ residues of U1 (Weber and Aebi 1988).

In contrast to metazoan introns, which typically deviate from the consensus, the sequence at the $5^{\prime}$ splice site in yeast introns is virtually invariant. Although similar to the mammalian consensus //GUPuAGU; Mount 1982), the yeast sequence differs by a transversion at the fourth position (/GUAUGU; Teem et al. 1984). The functional importance of these nucleotides in yeast splicing has been revealed by genetic perturbation (Jacquier et al. 1985; Parker and Guthrie 1985; Fouser and Friesen 1986; Vijayraghavan et al. 1986). Mutation at most positions strongly inhibits splicing and, unlike the situation in mammals, fails to activate cryptic splice sites (Gallwitz 1982; Pikielny et al. 1983; Newman et al. 1985). It was therefore anticipated that perfect complementarity between yeast $\mathrm{Ul}$ and the $5^{\prime}$ splice site would be an important precondition for $5^{\prime}$ splice site utiliza- 
tion. Because the yeast sequence differs from the mammalian consensus at the fourth position, it was specifically predicted that the $5^{\prime}$ end of yeast $\mathrm{U} 1$ would contain a compensating change ( $U 1-5 \mathrm{U}$ to $\mathrm{U} 1-5 \mathrm{~A}$ ) to allow basepairing at this position. Surprisingly, however, the first 10 nucleotides of yeast $\mathrm{U} 1$ are identical to human U1 [yeast U1 is the product of the SNR19 gene (Kretzner et al. 1987; Siliciano et al. 1987b/], precluding the possibility of Watson-Crick base-pairing at this position (Fig. 1A). This mismatch, along with a similar mismatch at position -2 , suggested that $5^{\prime}$ splice site recognition involves more than maximized base-pairing with U1 (Siliciano et al. 1987b).

We were thus prompted to undertake a detailed analysis of the contribution of base-pairing to splicing in Saccahromyces cerevisiae. In this work, we have addressed the impact of pairing potential at three positions in the proposed intermolecular helix. Three parameters were evaluated: the efficiency of each of the two steps in the splicing reaction, namely $5^{\prime}$ cleavage/lariat formation and $3^{\prime}$ cleavage/exon ligation, and the accuracy of $5^{\prime}$ splice site choice. The latter was made possible by the intriguing observation that mutation of the invariant
IVS-G5 to an A in the intron of several yeast genes activates an aberrant cleavage event near the original splice site (Jacquier et al. 1985; Parker and Guthrie 1985; Fouser and Friesen 1986). This mutant allows us to test the question of whether this incorrect $5^{\prime}$ site is a consequence of the intron mutation itself or of a novel pairing with yeast $U 1$.

\section{Results}

We have investigated the importance of base-pairing in the interaction between yeast $\mathrm{Ul}$ and the yeast $5^{\prime}$ splice site using a strategy of compensatory mutations (Fig. 2). Starting with point mutations in the $5^{\prime}$ splice site, we designed suppressor mutations in yeast $U 1$ that are predicted to restore base-pairing to the intron. As a substrate, we employed the actin-HIS4 fusion gene. Efficient removal of the actin intron from the fusion gene transcript is required for growth on the histidine precursor histidinol (abbreviated Hol; Parker and Guthrie 1985). Point mutations of the invariant G1 and G5 residues of the $5^{\prime}$ splice site have previously been intro-

\section{A. \\ C. \\ D.}

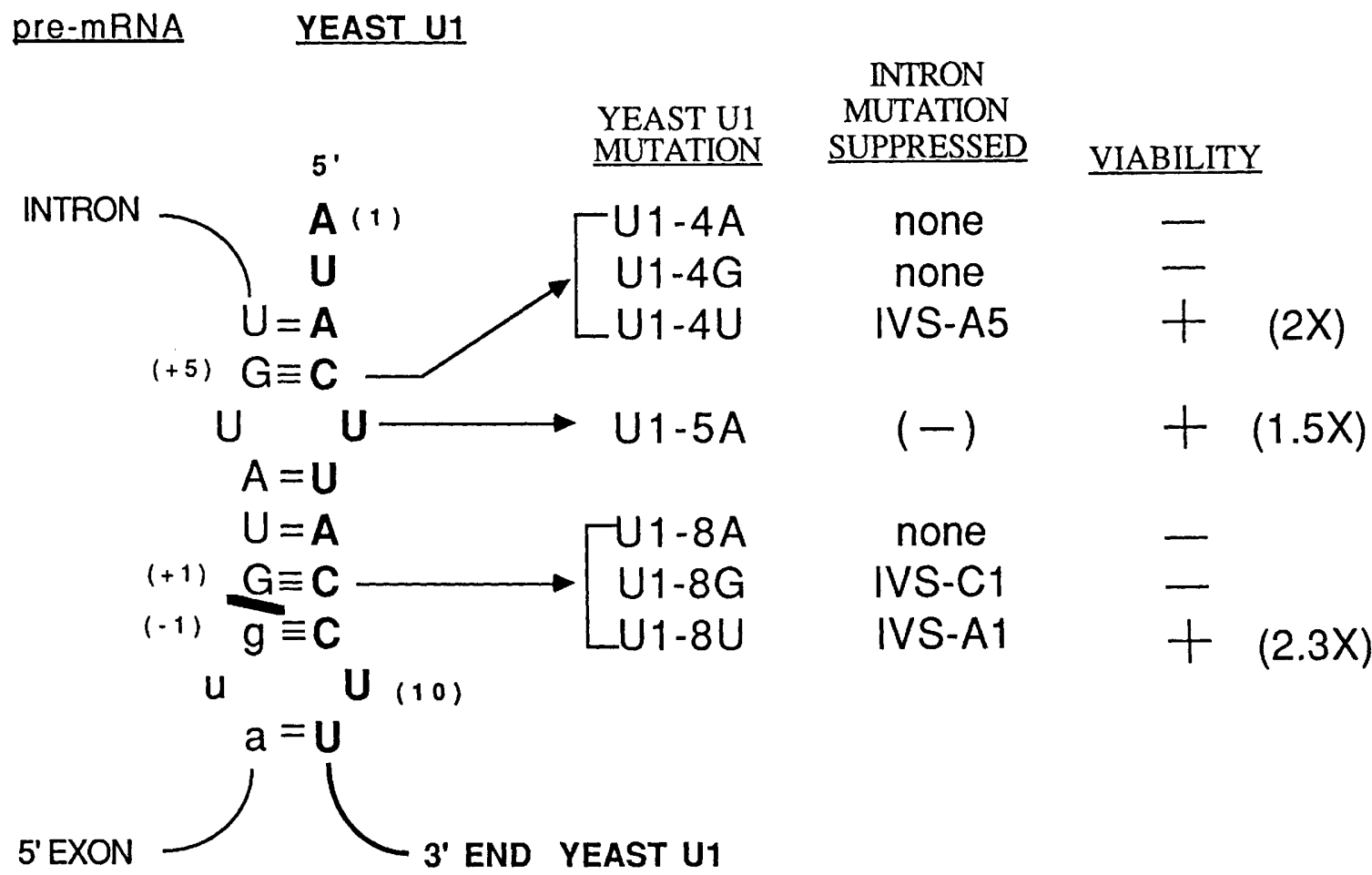

Figure 1. Base pairing between yeast $U 1$ and the yeast $5^{\prime}$ splice site consensus sequence. $(A \mid$ The proposed interaction between the yeast $5^{\prime}$ splice site consensus sequence and the first 11 nucleotides of $U 1$ is shown. The pre-mRNA is shown in lightface type running from the $5^{\prime}$ end (below) to the $3^{\prime}$ end (above). The heavy slash indicates the site of $5^{\prime}$ cleavage. Positions +1 to +6 are conserved to $>85 \%$ in yeast introns. Positions $-1,-2$, and -3 are drawn in lowercase letters because they are conserved $<60 \%$. The $5^{\prime} 11$ nucleotides of $U 1$ are shown in boldface type, nunning from the cap (above) toward the $3^{\prime}$ end (below). (B) The U1 point mutations used in this study are indicated. $(C)$ The intron mutations predicted to be suppressed by each U1 mutation are listed. Note that snR19-5A does not suppress any intron mutation; rather, it allows base-pairing at position 4 with wild-type introns. (D) The viability of the U1 point mutations in the absence of wild-type $\mathrm{Ul}$ is indicated, with the increase in doubling time caused by the mutation in parentheses. Cells were grown in rich media at $30^{\circ} \mathrm{C}$. 


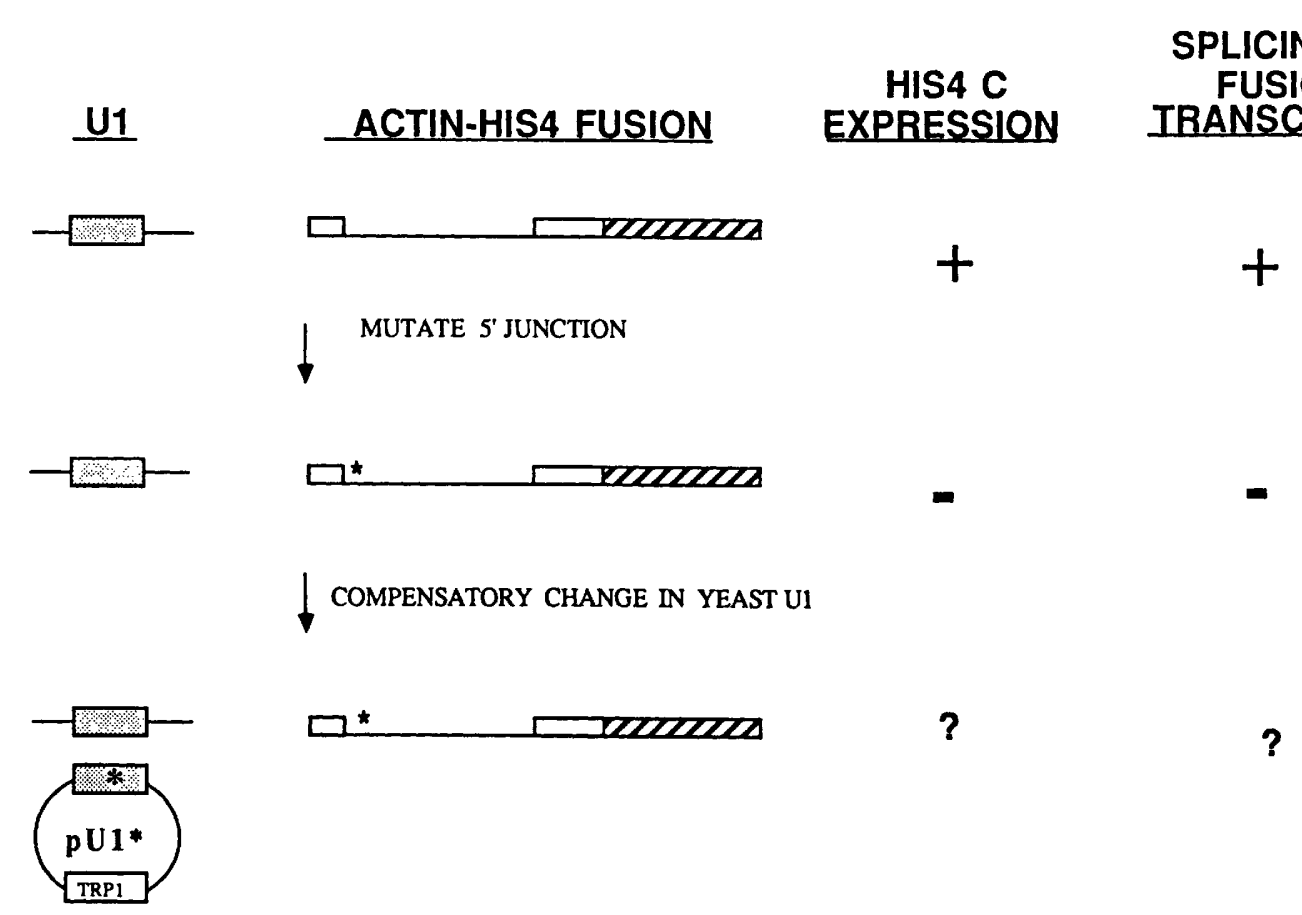

Figure 2. Strategy for testing base-pairing. Point mutations that block splicing have been introduced into the $5^{\prime}$ splice site of actinHIS4 fusion genes. Suppressor U1 mutants $\left(\mathrm{Ul}^{\circ}\right)$ are then introduced on TRP1-CEN4 plasmids, and their effect on splicing assayed by measuring growth on $\mathrm{Hol}$ and by primer extension. In these suppressor assays, wild-type $\mathrm{U} 1$ is provided from the wild-type copy of SNR19 at its normal chromosomal location. RNA sequencing using a labeled primer and reverse transcriptase (McPheeters et al. 1986) shows that the mutant Ul alleles are expressed at roughly wild-type levels (data not shown).

duced into the intron of the fusion gene and shown to interfere with splicing (Parker and Guthrie 1985; Vijayraghavan et al. 1986). Alterations in yeast Ul were designed to compensate for the intron mutations and were constructed in vitro by oligonucleotide-directed mutagenesis. These U1 mutants, carried on centromere-containing plasmids, were introduced by transformation into yeast strains containing mutant fusions. In this way, wild-type $U 1$ function is maintained by the normal copy of SNR19 in the chromosome. Suppression of the intron mutations was then assayed both biologically, by growth on Hol, and biochemically, by primer extension analysis of fusion transcripts.

\section{U1 base pairs with intron position 1 in the first step of splicing}

Compensatory changes in yeast $\mathrm{U} 1$ can suppress the inhibition of $5^{\prime}$ cleavage/lariat formation caused by mutations at position 1 of the $5^{\prime}$ splice site. Mutation of the invariant $G$ at the first position of the intron to an $A$ (IVS-Al, /GUAUGU to /ÂUAUGU; see Fig. 1) prevents splicing and does not allow cells containing IVS-A1 mutant fusion to grow on Hol (Vijayraghavan et al. 1986). Compared to wild-type transcripts, which are efficiently spliced to mature mRNA (Fig. 3A, lane 1), transcripts containing the IVS-Al mutation produce no mature mRNA but accumulate instead in the precursor/lariat intermediate ratio of $45: 55$ (Fig. 3A, lane 2). Introduc- tion of the U1-8U mutation, predicted to restore basepairing to IVS-Al, does not restore growth on $\mathrm{Hol}$ (data not shown), but suppression is witnessed by primer extension analysis of the IVS-Al transcripts (Fig. 3A, lane 3 ). In the presence of the U1-8U suppressor, $75 \%$ of the primary transcript completes the first step of splicing, accumulating as lariat intermediate. However, no mature mRNA is observed. The lack of mature mRNA corresponds with the failure of suppressed IVS-A1 to grow on Hol. No other yeast Ul mutation, either at position 8 or at other positions, increased the ability of IVS-Al to form lariat (data not shown).

A transversion at position 1 (IVS-Cl, /GUAUGU to /CUAUGU; see Fig. 1) inhibits splicing completely, and only full-length fusion transcript is observed (Fig. 3B, lane 3); fusions with the IVS-Cl mutation are $\mathrm{Hol}^{-}$(Vijayraghavan et al. 1986). Upon introduction of U1-8G, the predicted suppressor, cells containing the IVS- $\mathrm{Cl}$ fusion remain $\mathrm{Hol}^{-}$(data not shown). However, suppression of IVS- $\mathrm{Cl}$ is evidenced by primer extension analysis. Addition of the U1-8A mutant does not alter the primer extension pattern (Fig. 3B, lane 4), but in the presence of U1-8G, which should restore base-pairing to IVS-C1, $\sim 10 \%$ of the precursor is converted to lariat intermediate (lane 5). As with suppression of IVS-Al, no mature mRNA is detected, again, explaining the $\mathrm{Hol}^{-}$ phenotype. IVS-Cl suppression is also allele specific: Only the yeast Ul mutation predicted to restore basepairing improves splicing of IVS-Cl. 

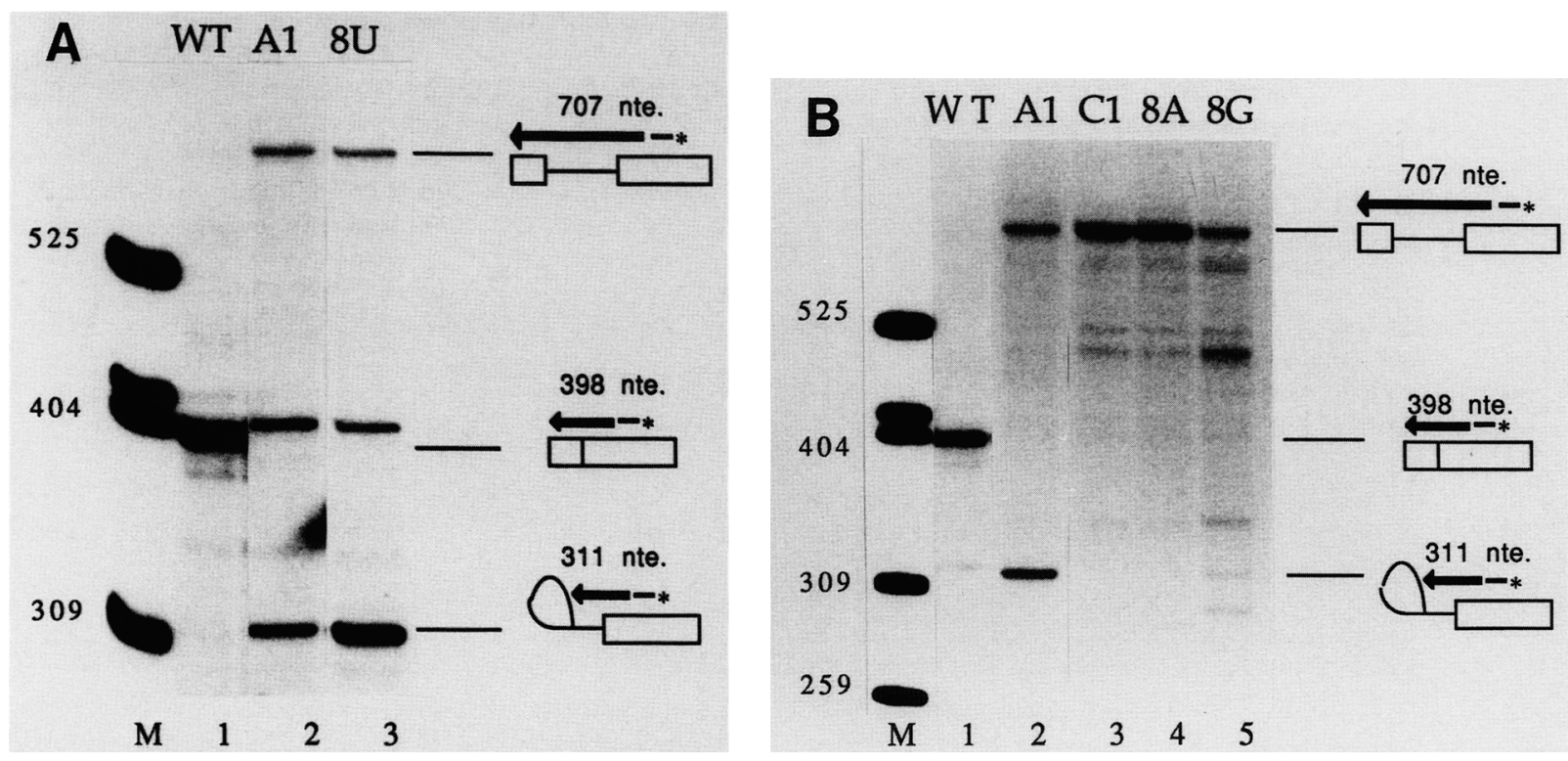

Figure 3. Primer extension analysis of position 1 mutants. (A) IVS-A1 primer extension using a primer (the 21 -mer) that anneals in the second exon. This primer (Vijayraghavan et al. 1986) and the primer extension protocol (Domdey et al. 1984) have been described. All primer extensions were performed at least twice using separate RNA preparations. The sizes of the primer extension products expected for precursor, mature, and lariat forms are cartooned. (M) End-labeled pBR325 HpaII fragments as markers; (lane 1) wild-type fusion to show the correct position of the primer extension product for mature mRNA; (lane 2) untransformed IVS-Al cells showing accumulation of precursor and lariat intermediates. The band just above 404 nucleotides is seen in some samples and not others (the same RNA is used in Fig. 3B, lane 2, where the artifactual band is not seen). This band results from a primer extension artifact and does not correspond to mature mRNA (J. Couto, pers. comm.); (lane 3) IVS-Al fusion transformed with the U1-8U suppressor. Because primer extension samples are difficult to compare directly, splicing efficiency is best compared by calculating the ratio of spliced products to unspliced precursor. These ratios were quantitated by densitometric scanning of light exposures of the autoradiograms. $(B)$ IVS-C1 primer extension using the 21-mer as above. (Lane 1) Wild-type fusion showing mature mRNA; (lane 2) IVS-Al fusion to show the positions of precursor and lariat; (lane 3) IVS-Cl fusion; (lane 4) IVS-C1 fusion transformed with U1-8A; (lane 5) IVS-Cl fusion transformed with U1-8G. The band at lariat is reproducibly seen in independent primer extension experiments using RNA from the IVS-C1 + U1-8G strain. Some primer extension products are darker in lane 5 than in other lanes; these bands are likely to be premature primer extension stops and are not reproducibly present. Therefore, the $10 \%$ lariat formation in this sample is calculated as the ratio of lariat to precursor, ignoring the other bands.

\section{U1 base pairs with intron position 5 in the first step of splicing}

Mutation of the fifth position of the intron to an A (IVSA5, /GUAUGU to /GUAUAUU; see Fig. 1) perturbs both the efficiency and fidelity of splicing (Parker and Guthrie 1985). Only $50 \%$ of IVS-A5 transcripts are processed into mature mRNA, allowing a weak $\mathrm{Hol}^{+}$phenotype (see Fig. 4A). Approximately $20 \%$ of IVS-A5 transcripts accumulate as lariat intermediate, whereas the remainder stay as unspliced precursor (Fig. 5A). In addition, $70 \%$ of the steady-state lariat produced is not cleaved at the correct $5^{\prime}$ splice site but at an aberrant site in the $5^{\prime}$ exon, five nucleotides upstream of the correct site. These aberrantly cleaved transcripts form lariat intermediates, as demonstrated by primer extension analyses and by debranching experiments (Fouser and Friesen 1986; Vijayraghavan et al. 1986). Lariat intermediates that have been cleaved at the aberrant site are not processed to mature message (Parker and Guthrie 1985).

Cells with the IVS-A5 mutation become fully $\mathrm{Hol}^{+}$ when the predicted suppressor, U1-4U, is introduced (Fig. 4A). The $\mathrm{Hol}^{+}$phenotype requires the U1-4U plasmid, as cells that spontaneously lose this plasmid revert to the normal level of IVS-A5 growth on Hol (Fig. 4A). Allele-specific suppression is also demonstrated by the Hol assay: No other yeast U1 point mutations allow growth (Fig. 4B).

Suppression is also demonstrated by the primer extension assay using a primer located in the second exon. The precursor/mature/lariat intermediate ratio of $30: 50: 20$ is unchanged in IVS-A5 cells transformed with yeast U1 mutants that do not compensate for IVS-A5 (Fig. 5A, lanes 1, 3, 4, and 5). Upon addition of U1-4U, the relative amount of precursor decreases twofold with a concomitant increase in the relative amounts of lariat intermediate and mature RNAs (Fig. $5 A$, lane 2). Suppression of IVS-A5 by U1-4U demonstrates base-pairing at this position.

\section{Suppression of IVS-A5 does not reduce aberrant cleavage}

Restoration of base-pairing between $\mathrm{U} 1$ and intron position 5 increases the utilization of the IVS-A5 splice site, but how does base-pairing affect the fidelity of $5^{\prime}$ cleavage in this mutant? To assess the effect of the 

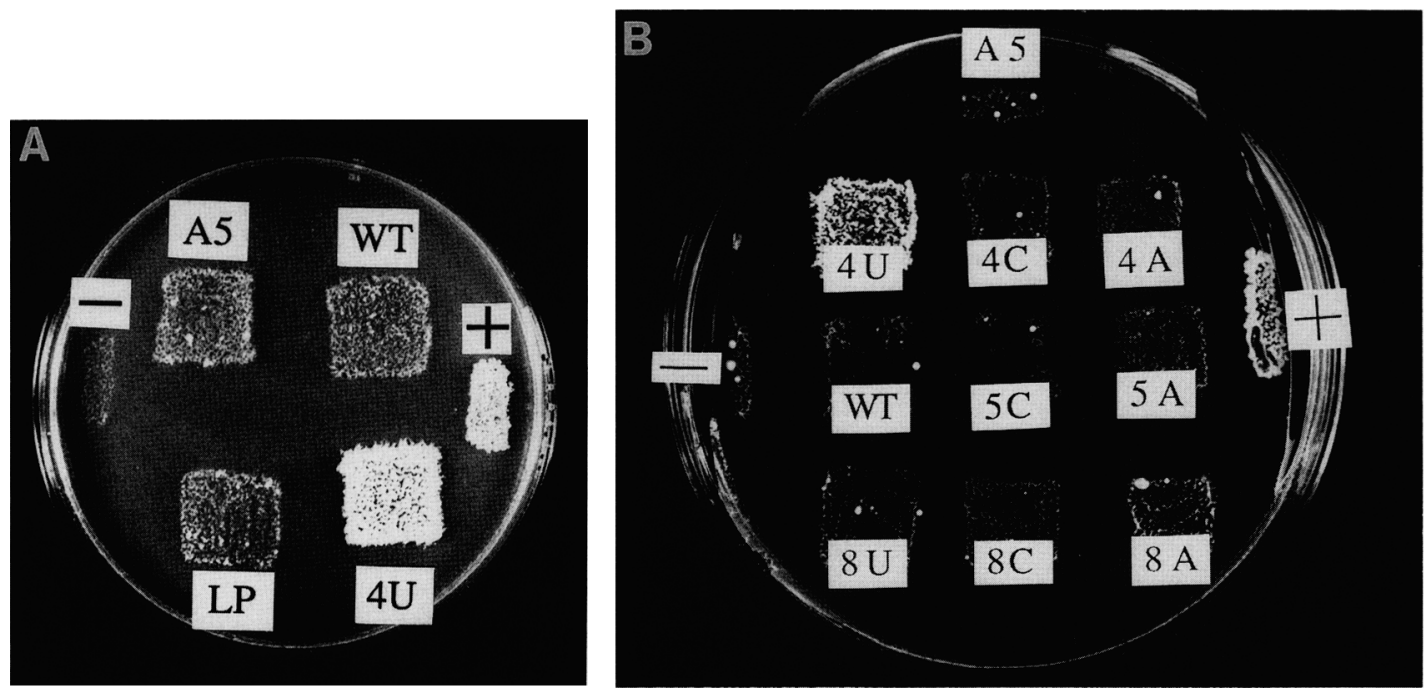

Figure 4. Suppression of the IVS-A5 mutation by Hol assay. $(A)$ The Hol phenotype of IVS-A5 is suppressed by the compensatory change in U1. Yeast cells growing on a Hol plate are untransformed IVS-A5 fusion cells (A5); IVS-A5 cells transformed with wild-type U1 (WT); IVS-A5 cells transformed with Ul-4U (4U); IVS-A5 cells transformed with Ul-4U, that have lost the U1 plasmid (LP). $(+\mid$ Wild-type fusion; $(-)$ no fusion. (B) Allele specificity of IVS-A5 suppression. Only the compensatory change in U1 suppresses the IVS-A5 Hol phenotype. IVS-A5 cells on Hol media, transformed with various U1 point mutations, as above.

U1-4U suppressor on the IVS-A5 aberrant cut, we used another primer (22-mer), which anneals to the intron between the branch site and the 5 ' splice site. This primer gives extension products for precursor and lariat intermediates only and can be used to determine the precise site of $5^{\prime}$ cleavage in lariat forms (by extending around the lariat and running into the branch site from the $5^{\prime}$ junction site; see diagram in Fig. 5B|. As assayed with this primer, no yeast Ul mutation tested changes the amount of incorrect lariat intermediate except U1-4U (Fig. 5B). Surprisingly, however, the suppressor U1 appears to increase the amount of incorrect lariat intermediate. The U1-4U allele by itself does not preferentially increase the use of the aberrant cleavage site, as this al-

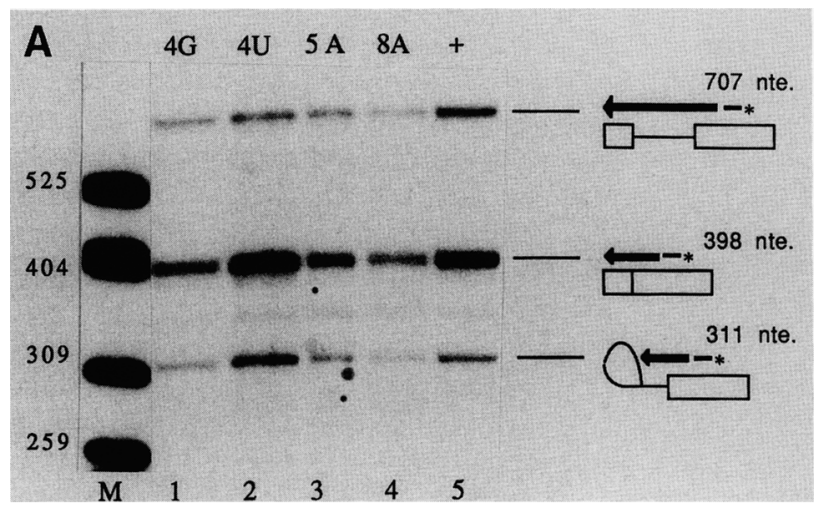

Figure 5. Primer extension analysis of IVS-A5. $(A)$ IVS-A5 primer extension using the 21-mer as above. (Lane 1) IVS-A5 transformed with U1-4G; (lane 2) IVS-A5 transformed with U1-4U; (lane 3) IVS-A5 transformed with U1-5A; (lane 4) IVS-A5 transformed with U1-8A; (lane 5) IVS-A5 transformed with wild-type U1. (B) Primer extension to show IVS-A5 cut site. These primer extensions use the 22-mer primer, which anneals between the branch site and the 5' junction. This primer gives extension products (as doublets) for precursor (177 nucleotides) and for correctly cleaved (45 nucleotides) and incorrectly cleaved (50 nucleotides) lariat. (Lane 1) IVS-A5 transformed with U1-4A; (lane 2) IVS-A5 transformed with U1-4G; (lane 3) IVS-A5 transformed with U1-4U; (lane 4) IVS-A5 transformed with U1-5A; (lane 5) IVS-A5 transformed with U1-8A; (lane 6) IVS-A5 transformed with wild-type U1.

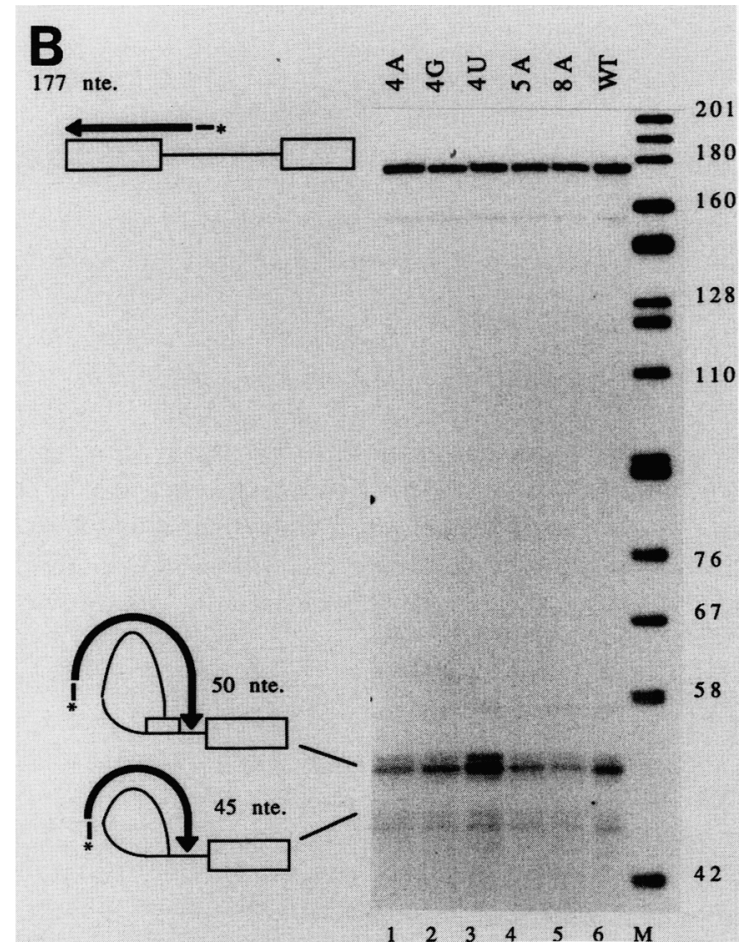


lele does not cause aberrant cleavage of a wild-type fusion (data not shown).

\section{Effect of base-pairing with U1 on the second step of splicing}

Experiments with both mammalian and yeast introns show that the sequence at the $5^{\prime}$ splice site plays an important role in $3^{\prime}$ cleavage/exon ligation. This is demonstrated by the fact that certain mutations at the first two positions of mammalian introns still allow the first step, albeit inefficiently, but completely inhibit the second step of splicing (reviewed in Aebi and Weissmann 1987). Similar observations have been made for position $1 \mathrm{mu}$ tations in yeast (Newman et al. 1985; Fouser and Friesen 1986; Vijayraghavan et al. 1986). These observations did not resolve whether this inhibition of $3^{\prime}$ cleavage/exon ligation was caused by a requirement for specific residues at the $5^{\prime}$ splice site, by failure to base pair with U1, or both. Using compensatory base changes between U1 and the 5 ' splice site, we can address these questions. In the presence of their cognate U1 suppressor alleles, both IVS-A1 (Fig. 3A, lane 3) and IVS-C1 (Fig. 3B, lane 5) are able to form lariat. However, neither lariat is converted to mature mRNA, even though the suppressor U1 alleles restore complementarity to the intron position 1 mutations (Fig. 3). This observation explains the failure of IVS-Al and IVS-Cl to grow on $\mathrm{Hol}$ in the presence of their U1 suppressor alleles; no mature mRNA is formed. Thus, we conclude that restoration of the ability to base pair with U1 is not sufficient to restore activity in the second step of splicing to intron position 1 mutants.

\section{SNR19 is an essential gene}

We next tested the point mutations generated above for the ability to function in the splicing of wild-type introns. First, the single copy SNR19 gene (Kretzner et al. 1987; Siliciano et al. 1987b/ was disrupted to test the prediction that U1, like the other yeast Sm-snRNA analogs (Ares 1986; Patterson and Guthrie 1987; Siliciano et al. 1987a; Brow and Guthrie 1988), is an essential gene. The disruption allele (snr19::LYS2, see Fig. 6A) was used to replace one copy of $S N R 19$ in a wild-type diploid, and the integration was confirmed by Southern analysis (Fig. 6B). Upon sporulation, this heterozygous diploid gives rise to only two viable spores per tetrad, indicating the disruption of an essential gene (Fig. 6C). No Lys ${ }^{+}$spores are recovered, mapping the lethality to the snr19:: LYS 2 construction. Lys ${ }^{+}$spores can be recovered if the heterozygous diploid is transformed with a plasmid carrying wild-type $S N R 19$ prior to sporulation, demonstrating that the lethal phenotype can be complemented in trans data not shown).

\section{Some yeast U1 point mutants are viable}

The point mutants generated above were transformed into the diploid strain heterozygous for the snr $19:$ :LYS2 disruption and tested for complementation. Tetrad anal- ysis demonstrates that haploid cells containing either U1-4U (the IVS-A5 suppressor) or U1-8U (the IVS-A1 suppressor) are viable in the absence of wild-type U1; all other mutations at positions 4 and 8 confer lethal phenotypes (see Fig. 1D). The U1-4U and U1-8U alleles are not completely wild type for U1 function, however, as both mutants exhibit significantly increased doubling times compared to sister spores with a wild-type copy of SNR19 (Fig. 1D). Growth at high temperatures $\left(37^{\circ} \mathrm{C}\right)$ exaggerates this effect. Interestingly, both these yeast $\mathrm{Ul}$ alleles would allow G-U pairing with their respective partners in the intron.

\section{Base-pairing is not observed at IVS position 4}

As pointed out in the introductory paragraphs, the conservation of the $U$ at position 5 of yeast $U 1$ is puzzling in that this residue is unable to form a canonical base pair with the conserved intron nucleotide IVS-4U. One pos-
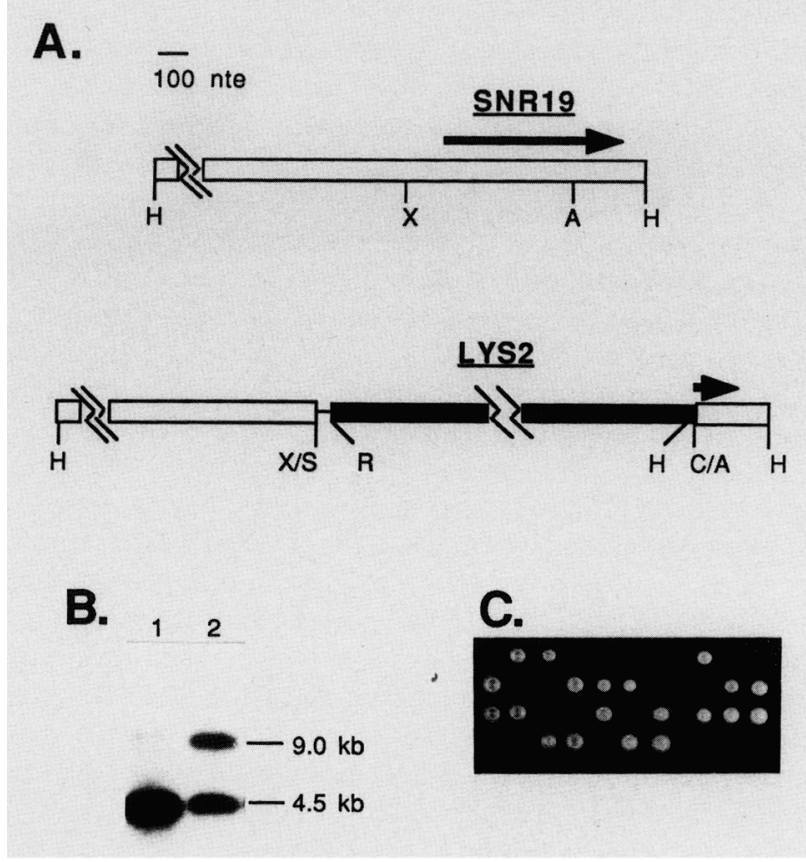

Figure 6. Yeast $U 1$ performs an essential function. (A) The wild-type SNR19 gene (above) and the $\operatorname{sr} 19::$ LYS2 disruption allele (below), which deletes the $5^{\prime}$ two thirds of SNR19 and replaces it with the yeast LYS2 gene are diagramed (the construction is described in Methods). The SNR19-coding region is depicted by the arrow. The LYS2 gene is represented by a dark box, and a short piece of the BlueScript polylinker is shown as a thin line. The drawing of the snr19:: LYS2 construction is not to scale. Restriction sites: (H) HindIII; (X)XmnI; (A) AsuII; (S) SmaI; (R) EcoRI; (C) ClaI. (B) The integration of a single copy of the disruption allele at the SNR 19 locus was confirmed by Southern blot. DNA isolated from the TR1 parent (lane 1) and disrupted (lane 2) diploids was digested with HindIII, Southern blotted, and probed for SNR19. The wild-type 4.5-kb HindIII fragment is seen in both cells, but the disrupted cells also contain a band $4.5 \mathrm{~kb}$ larger due to the disruption allele. The $300-$ nucleotide band corresponding to the $3^{\prime}$ terminal HindIII fragment of the disrupted allele is not seen here. $(C)$ Tetrad dissection to demonstrate the SNR19 encodes an essential function. 
sible reason for the conservation of U1-5U is that it is required for other interactions. The helix bulge caused by the U-U mismatch might also be a structural signal in 5' splice site recognition. An alternative hypothesis is that increased complementarity would have an inhibitory effect on splicing efficiency. To test these models, we constructed U1-5A, which has the potential to base pair with the yeast $5^{\prime}$ junction sequence at this position (see Fig. 1).

When U1-5A is introduced into a cell in the presence of wild-type Ul, it causes no dominant inhibition of growth. Moreover, when the ability to complement the snr19:: LYS2 disruption allele is tested (i.e., in the absence of wild-type U1), cells with only U1-5A are fully viable, albeit with a $50 \%$ increase in doubling time (Fig. $1 D \mid$. This surprising result demonstrates that the conserved $U$ residue at nucleotide 5 in yeast $U 1$ is not essential and that the U-U mismatch between the yeast RNAs is not obligatory. In addition, base-pairing potential with intron position 4 is not strongly detrimental to splicing efficiency. Although the increase in doubling time does suggest some inhibition of processing, we have not been able to identify specific transcripts for which splicing has become rate limiting in these cells.

Of particular interest was the pre-mRNA for ribosomal protein $R P L 32$, whose 5 ' splice site deviates from the consensus by two transversions (/GUCAGU; Dabeva and Warner 1987). Although this transcript would thus be predicted to be spliced inefficiently, primer extension assays reveal only a very low level of unspliced $R P L 32$ precursor in wild-type cells (Dabeva et al. 1986; T. Simmons, unpubl.). We conjectured that the destabilization caused by the mismatch at position 3 is countered by the ability to base pair with wild-type yeast Ul at position 4 . If this were the case, the base pair at intron position 4 could not form in cells with only U1-5A, and splicing of RPL32 should be less efficient. By primer extension and Northern analysis, however, we find no increase in the ratio of precursor/mature RPL32 RNA in these cells (data not shown). Therefore, base-pairing of intron position 4 with $U 1$ does not appear to be required for efficient splicing of RPL32.

\section{Discussion}

U1 base pairs with the $5^{\prime}$ splice site at some but not all positions

Our results show that base-pairing of intron positions 1 and 5 with $U 1$ is directly involved in the first step of splicing. This observation is consistent with experiments in mammalian systems, which showed that the efficiency of splice site use increased if the complementarity to U1 was restored at intron position 5 (Zhuang and Weiner 1986; base-pairing at position 1 was not tested in these experiments).

However, not all positions in the $5^{\prime}$ splice site appear to be involved in essential base-pairing interactions with U1. Evidence for base-pairing with intron position 3 , the only other compensatory change tested in the mammalian in vitro system, was very weak at best (Zhuang and
Weiner 1986). Position 4 in yeast introns is normally unpaired, and our analysis of an atypical transcript (RPL32) predicted to be dependent on this complementarity suggests that either this base pair does not form or that the improved stability of the intermolecular helix is not rate determining. Because the RPL32 $5^{\prime}$ splice site cannot pair with U1-5A at positions 3 or 4, yet is still spliced at normal efficiency, base-pairing at position 3 is also not essential. Finally, due to the poor conservation of positions -1 to -3 in yeast transcripts (see Fig. 1), ranging from $58 \%$ to $42 \%$ (Teem et al. 1984), few introns are able to base pair with $\mathrm{Ul}$ at each of these positions. In summary, the maximum match to $\mathrm{U} 1$ in yeast usually allows $7 \mathrm{bp}$ within the nine nucleotides spanning the $5^{\prime}$ splice site, whereas the average splice site pairs at only five positions. It is likely that each position in the $5^{\prime}$ splice site has a characteristic contribution to the base-pairing interaction, ranging from critical (e.g., position 1) to unimportant (position 4).

Few mammalian introns fit the $5^{\prime}$ splice site consensus sequence perfectly, although the average has the potential to match at seven out of nine positions (Mount et al. 1983). Although positions 1, 2, and 5 are most highly conserved in mammals, all except position 1 can vary. Mismatch may be an important factor in regulating splice site utilization, although potential complementarity to U1 does not always correlate with efficiency of $5^{\prime}$ splice site use (Zhuang et al. 1987). In fact, Nelson and Green (1988) have shown that potential 5' splice sites that are not used still bind U1 snRNPs, suggesting that complementarity with $\mathrm{Ul}$ is not the sole determinant in $5^{\prime}$ splice site selection.

\section{Restoration of base-pairing with U1 increases the efficiency of the first step only}

It has been observed previously in mammalian (Aebi et al. 1986) and yeast systems (Newman et al. 1985; Vijayraghavan et al. 1986) that the sequence requirements for the second step of splicing appear to be more stringent, as some $5^{\prime}$ splice site mutants are partially active in the first step (e.g., IVS-A1) but totally inactive in the second. However, it was not clear whether the failure to undergo 3 'cleavage/exon ligation was due to the sequence changes themselves or to the failure to allow stable base-pairing with U1. We can now argue in favor of the former hypothesis because neither IVS-Al nor IVS-Cl lariat intermediate is able to undergo the second step of splicing at a detectable level when suppressed by compensatory changes in U1. It is unclear why the particular structure of the branch, which does not appear to participate directly in $3^{\prime}$ cleavage/exon ligation, should be essential for that step. An interesting suggestion is that this requirement is indicative of a proofreading mechanism, employed to abort accidental missplices.

\section{Conservation of $U 1$}

The viability of strains containing Ul with point mutations in 3 of the first 10 nucleotides (U1-4U, U1-8U, and 
U1-5A) was unexpected in light of the evolutionary invariance of this sequence. Nonetheless, the fact that those changes that would allow G-U base pairs to form at intron positions 1 and 5 are viable and the other changes are not further supports the role of base-pairing between $\mathrm{U} 1$ and the $5^{\prime}$ splice site at these positions.

Particularly curious is the conservation of U1 nucleotide 5, which regiments a mismatch at intron position 4 Mutation to U1-5A causes only a $50 \%$ increase in doubling time. This increased doubling time might be due to formation of too many base pairs with wild-type introns; e.g., extensive base-pairing might slow release of $\mathrm{U} 1$, thereby slowing the splicing reaction. Alternatively, splicing of an intron with an unusual $5^{\prime}$ splice site, which utilizes base-pairing at this position, may be impaired. However, examination of transcripts with both wild type $\{C Y H 2\}$ and position 4 complementary (RPL32) $5^{\prime}$ splice sites has not revealed any splicing defect in these cells.

The precise location of the $5^{\prime}$ cut is not determined solely by base pairing with $U 1$

Actin introns with the IVS-A5 mutation are cleaved at an aberrant site five nucleotides upstream of the correct site (Parker and Guthrie 1985); the IVS-C5 mutation causes the same effect (Fouser and Friesen 1986). Although the compensatory change in U1 (U1-4U) increases overall splicing efficiency, it does not suppress the IVS-A5 aberrant cleavage. As discussed in the introductory paragraphs, experiments in mammalian in vitro systems have recently led to the suggestion that the site of $5^{\prime}$ cleavage is determined by base-pairing with sequences in U1 (Aebi et al. 1987; Weber and Aebi 1988). Based on a strict extrapolation from this model, we would have predicted that the IVS-A5 aberrant cleavage is a consequence of $\mathrm{U} 1$ selecting an alternative basepairing (Fig. 7A), five nucleotides upstream of the normal interaction, placing the site of aberrant cleavage opposite the $\mathrm{C} 8-\mathrm{C} 9$ residues of $\mathrm{U} 1$. However, in the actin sequence, this pairing (Fig. 7A) is very weak and seems unlikely to occur in vivo.

Furthermore, increased use of the aberrant cut site in the presence of the suppressor cannot be explained by this model. Because the compensatory change in yeast U1 (Fig. 7B) restores a wild-type set of base pairs to the 'normal' interaction while weakening the 'aberrant' pairing, a U1 mutant that restores base-pairing to IVS-A5 should also reduce use of the aberrant cleavage site. We conclude that the increased activity at this $5^{\prime}$ splice site is due to the restored complementarity with $\mathrm{U} 1$, which allows a more stable intermolecular helix to form and thereby increases splicing efficiency. The persistence of the aberrant cut, however, demonstrates that pairing per se does not dictate the location of $5^{\prime}$ cleavage site.

The IVS-A5 mutation also causes aberrant cleavage when present in the intron of the ribosomal protein $R P 51 \mathrm{~A}$, in this case activating cleavage three nucleotides upstream of the correct site (Jacquier et al. 1985).
This sequence, 5'-UAACAAA:AUG/GUAUAUUAAU$3^{\prime}$ (from -10 to +10 ; / indicates the correct cleavage site: indicates the aberrant cut site caused in RP51A by IVS-A5) differs from the actin sequence and, like actin, has no clear complementarity that would put $\mathrm{C} 8-\mathrm{C} 9$ of U1 across from the aberrant cut site. Thus, the incorrect cleavage events are not constrained to occur at specific nucleotides or at a fixed distance from intron position 5 . That the IVS-A5 mutation in another intron also causes aberrant cleavage strongly supports the argument that position 5 helps to localize the $5^{\prime}$ cut.

Whether the differences between the yeast results presented here and those from the mammalian system (Weber and Aebi 1988) are due to different assay systems or to more fundamental differences in 5 ' splice site selection remains to be tested. It is clear, however, that a mechanistic understanding of $5^{\prime}$ splice site selection in yeast is lacking. We can now use genetic analysis to address this question.

\section{Methods}

\section{Materials}

Restriction enzymes were purchased from New England Biolabs, BRL, Boehringer Mannheim, USB, and Toyobo. T4 polynucleotide kinase was obtained from Pharmacia, and reverse transcriptase from Life Sciences. Nick translations were performed using the Amersham Nick Translation Kit. [ $\left.{ }^{32} \mathrm{P}\right] \mathrm{ATP}$ was purchased from ICN, and the Muta-Gene enzyme refill kit from Bio-Rad.

\section{Oligomutagenesis}

Point mutations in yeast U1 were made using the $d u t^{-} u^{-}$ technique of Kunkel et al. (1987), as modified by Evnin and Craik (1988). A 1.6-kb HindIII fragment containing the SNR19coding region was subcloned into Bluescript + (Stratagene). Single-stranded, uracil-containing template was prepared and mutagenized as described (Evnin and Craik 1988). Resultant plasmids were screened for mutants by dideoxy sequencing (Sanger et al. 1977) using the 19G primer (5'-CAGTAGGACTTCTTGATC-3'), which anneals from +43 to +26 . For each position mutagenized, a single triply degenerate oligonucleotide was used to make all three possible changes in one mutagenesis. Mutagenesis efficiency varied between $50 \%$ and $80 \%$. Mutant SNR19 fragments were subcloned into the yeast vector pSE-358 (S. Ellidge, pers. comm.), which carries CEN IV, as well as the TRP1 gene, as a selectable marker. All mutants were resequenced after subcloning into pSE358 to confirm the constructions.

\section{Construction of the SNR19 disruption allele}

The snr19:: LYS2 allele deletes the $5^{\prime}$ two thirds of the coding region (from the $X m n I$ site at -82 in the $5^{\prime}$ noncoding region to the AsuIl site at +396 ) and replaces them with the yeast LYS2 gene. This allele was constructed by assembling the desired fragments in the polylinker of Bluescript+ (Stratagene). Starting with a $1.6-\mathrm{kb}$ fragment in the HindIII site, the $5^{\prime}$ portion of the fragment was removed by digestion with EcoRI (in the polylinker) and AsuII (in SNR19). Next, a 5 -kb fragment containing the LYS2 gene was added as an EcoRI-ClaI frag- 
Figure 7. Base pairing of the IVS-A5 intron. $|A|$ Pairings between IVS-A 5 and wild-type yeast U1. The sequence of the actin intron with the IVS-A5 mutation is shown in lightface type from $5^{\prime}$ on the left to $3^{\prime}$ on the right. The heavy slash marks the position of the normal 5 ' cleavage; the downward arrow shows the position of the IVS-A5 aberrant cleavage. The mutant nucleotide IVS-A5 is shown in hollow type. The normal Ul pairing is shown above the intron sequence, and a potential pairing, which would select the IVS-A5 cleavage site, is shown below the intron. This pairing is selected because it places the $\mathrm{C} 8-\mathrm{C} 9$ residues of $\mathrm{U} 1$ across from the aberrant cut, as required by strict extrapolation from the mammalian data. Alternative pairings, which may have greater stability, are possible but would require bulging a nucleotide on one side of the helix only, would pair nucleotides of U1 that are not normally involved in base-pairing with the intron, or would put $\mathrm{C} 8-\mathrm{C} 9$ in other locations. These pairings are not considered here because they would represent a significant deviation from the normal configuration of $\mathrm{Ul}$ basepairing. (B) Pairings between IVS-A5 and U1-4U. The IVS-A5 actin intron is drawn as in $A$ and is shown paired with U1-4U. As above, the normal pairing is drawn above the intron, and the same alternative pairing in $A$, shown below. The $\mathrm{U} 1-4 \mathrm{U}$ mutation is shown in hollow type.
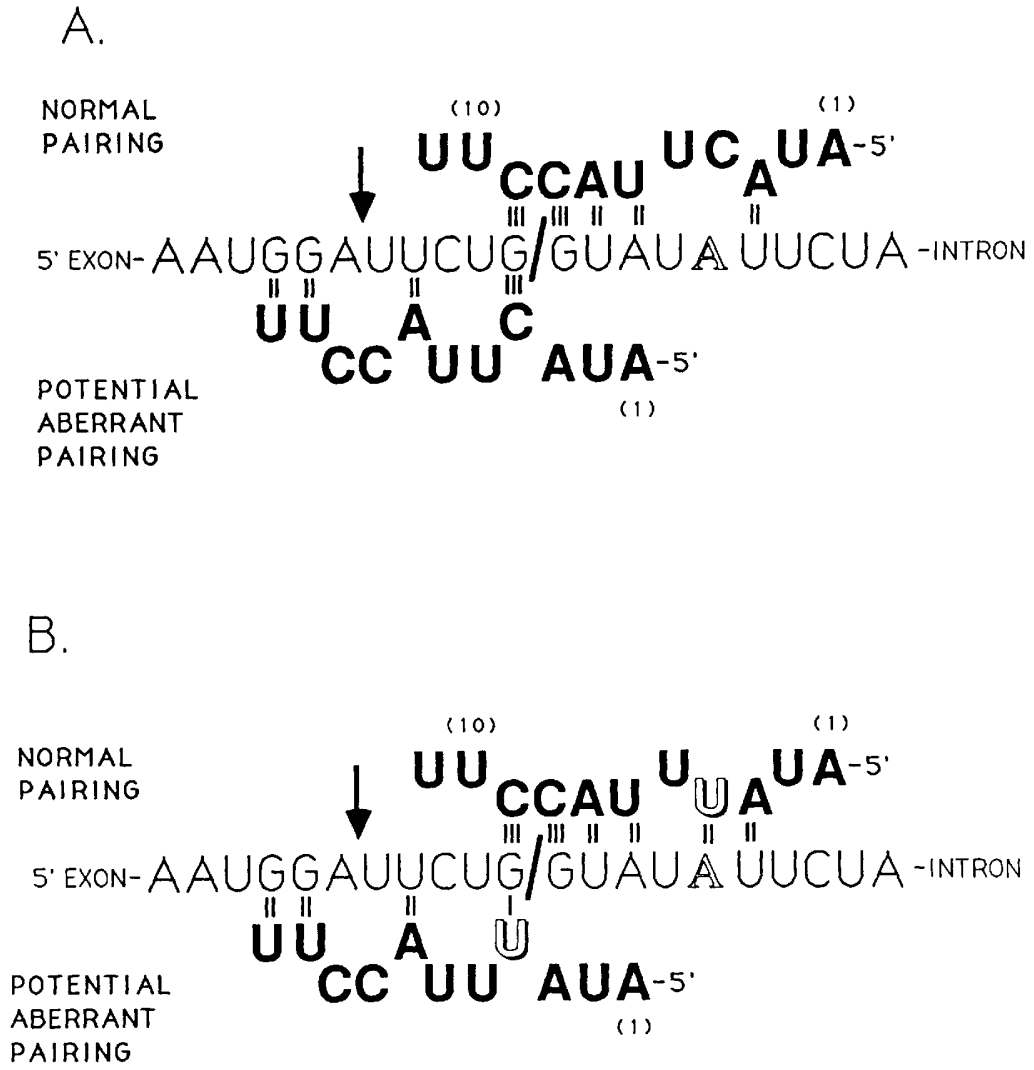

ment. Finally, an $X b a I-X m n I$ fragment from the starting plasmid (the $X b a I$ site is in the polylinker upstream of the SNR19 insert, the XmnI site is between the SNR19 TATA box and the transcription start site) containing the SNR19 5'flanking sequences was added. For integration into yeast, the disruption allele was cut out of the plasmid with XbaI and ClaI.

\section{Yeast strains}

The IVS-A5 mutant fusion is integrated in strain FC2-12B (MATa trp1 leu2 ura3 his4 HOL1; Parker and Guthrie 1985), all other fusions described were integrated into strain YJC59 (MATa ade2 can1 trp1 leu2 ura3 his4 HOL1; Couto et al. 1987). The SNR19 gene disruption was integrated into strain TRl (a/ $\alpha$ trp1/trp1 lys2/lys2 his3/his3 ura3/ura3) by the method of Rothstein (1983). The resulting strain, TR1-19 DD.1, was sporulated, and tetrads dissected to test the lethality of SNR19 disruption. This strain was also transformed with wild-type and mutant yeast $\mathrm{Ul}$ alleles to test complementation.

\section{Yeast transformation and RNA analysis}

Yeast transformations were performed by the method of Ito et al. (1983). Hol phenotypes were checked on Hol media, as described (Parker and Guthrie 1985). RNA was extracted by the guanidinium/hot phenol method (Wise et al. 1983) and analyzed by primer extension according to Domdey et al. (1984). For each sample, $20 \mu \mathrm{g}$ of total RNA was used. To assay expression of the mutant U1 alleles, the same RNA was sequenced using a labeled primer and reverse transcriptase in the presence of dideoxynucleotide triphosphates (McPheeters et al. 1986).

\section{Acknowledgments}

We thank Bertrand Seraphin for communication of results prior to publication. We thank Luke Evnin for help with the oligomutagenesis; Roy Parker, Bruce Patterson, and Trey Simmons for helpful discussions; Lucita Esperas for peerless technical assistance; and Judy Piccini for help in preparation of the manuscript. We are grateful to Roy Parker, Joe Couto, Michele Haltiner-Jones, Bruce Patterson, Trey Simmons, and Dan Frank for comments on the manuscript. This work was supported by National Institutes of Health grant GM-21119 and National Science Foundation grant DCB8603926 to C.G. P.G.S. was supported by a Damon Runyon-Walter Winchell postdoctoral fellowship (DRG-872).

\section{References}

Aebi, M., H. Honig, R.A. Padgett, J. Reiser, and C. Weissmann. 1986. Sequence requirements for splicing of higher eukaryotic nuclear pre-mRNA. Cell 47: 555-565.

Aebi, M., H. Hornig, and C. Weissmann. 1987. 5' cleavage site in eukaryotic pre-mRNA splicing is determined by the overall $5^{\prime}$ splice region, not by the conserved 5' GU. Cell 50: $237-246$.

Aebi, M. and C. Weissmann. 1987. Precision and orderliness in splicing. Trends Genet. 4: 102-107.

Ares, M. 1986. U2 RNA from yeast is unexpectedly large and contains homology to vertebrate $\mathrm{U} 4, \mathrm{U} 5$, and $\mathrm{U} 6$ small $\mathrm{nu}$ clear RNAs. Cell 47: 49-59.

Black, D.L., B. Chabot, and J.A. Steitz. 1985. U2 as well as U1 small nuclear ribonucleoproteins are involved in premessenger RNA splicing. Cell 42: 737-750. 
Brow, D.A. and C. Guthrie. 1988. Spliceosomal RNA U6 is remarkably conserved from yeast to humans. Nature 334: 213-218.

Chabot, B., D.L. Black, D.M. LeMaster, and J.A. Steitz. 1985. The $3^{\prime}$ splice site of pre-messenger RNA is recognized by a small nuclear ribonucleoprotein. Science 230: 1344-1349.

Couto, J.R., J. Tamm, R. Parker, and C. Guthrie. 1987. A transacting suppressor restores splicing of a yeast intron with a branch point mutation. Genes Dev. 1: 445-455.

Dabeva, M.D., M.A. Post-Beittenmiller, J.R. Warner. 1986. Autogenous regulation of splicing of the transcript of a yeast ribosomal protein gene. Proc. Natl. Acad. Sci. 83: 58545857.

Dabeva, M.D. and J.R. Warner. 1987. The yeast ribosomal protein L32 and its gene. J. Biol. Chem. 262: 16055-16059.

Domdey, H., B. Apostol, R.-J. Lin, A. Newman, E. Brody, and J. Abelson. 1984. Lariat structures are in vivo intermediates in yeast pre-mRNA splicing. Cell 39: 611-621.

Evnin, L.B. and C.S. Craik. 1988. Development of an efficient method for generating and screening active trypsin and trypsin variants. Ann. N.Y. Acad. Sci. (in press).

Fouser, L.A. and J.D. Friesen. 1986. Mutations in a yeast intron demonstrate the importance of specific conserved nucleotides for the two stages of nuclear mRNA splicing. Cell 45: $81-93$.

Gallwitz, D. 1982. Construction of a yeast actin gene intron deletion mutant that is defective in splicing and leads to the accumulation of precursor RNA in transformed yeast cells. Proc. Natl. Acad. Sci. 79: 3493-3497.

Ito, H., Y. Fukuda, K. Murata, and A. Kimura. 1983. Transformation of intact yeast cells treated with alkali cations. $J$. Bacteriol. 153: 163-168.

Jacquier, A., J.R. Rodriguez, and M. Rosbash. 1985. A quantitative analysis of the effects of $5^{\prime}$ junction and TACTAAC box mutants and mutant combinations on yeast mRNA splicing. Cell 43: 423-430.

Krainer, A.R. and T. Maniatis. 1985. Multiple factors including the small nuclear ribonucleoproteins $\mathrm{U} 1$ and $\mathrm{U} 2$ are necessary for pre-mRNA splicing in vitro. Cell 42: 725-736.

Kramer, A., W. Keller, B. Appel, and R. Luhrmann. 1984. The $5^{\prime}$ terminus of the RNA moiety of Ul small nuclear ribonucleoprotein particles is required for the splicing of messenger RNA precursors. Cell 38: 299-307.

Kretzner, L., B.C. Rymond, and M. Rosbash. 1987. S. cerevisiae U1 RNA is large and has limited primary sequence homology to metazoan Ul snRNA. Cell 50: 593-602.

Kunkel, T.A., J.D. Roberts, and R.A. Zakour. 1987. Rapid and efficient site-specific mutagenesis without phenotypic selection. Methods Enzymol. 154: 367-382.

Lerner, M.R., J.A. Boyle, S.M. Mount, S.L. Wolin, and J.A. Steitz. 1980. Are snRNPs involved in splicing? Nature 283: $220-224$.

McPheeters, D.S., A. Christensen, E.T. Young, G. Stormo, and L. Gold. 1986. Translational regulation of expression of the bacteriophage T4 lysozyme gene. Nucleic Acids Res. 14: 5813-5826.

Mount, S.M. 1982. A catalogue of splice junction sequences. Nucleic Acids Res. 10: 459-472.

Mount, S.M., I. Pettersson, M. Hinterberger, A. Karmas, and J.A. Steitz. 1983. The U1 small nuclear RNA-protein complex selectively binds a $5^{\prime}$ splice site in vitro. Cell 33: 509518.

Nelson, K.K. and M.R. Green. 1988. Splice site selection and ribonucleoprotein complex assembly during in vitro premRNA splicing. Genes Dev. 2: 319-329.
Newman, A.J., R.-J. Lin, S.-C. Cheng, and J. Abelson. 1985. Molecular consequences of specific intron mutations on yeast mRNA splicing in vivo and in vitro. Cell 42: 335-344.

Parker, R. and C. Guthrie. 1985. A point mutation in the conserved hexanucleotide at a yeast $5^{\prime}$ splice junction uncouples recognition, cleavage, and ligation. Cell 41: 107118.

Patterson, B. and C. Guthrie. 1987. An essential yeast snRNA with a U5-like domain is required for splicing in vivo. Cell 49: $613-624$.

Pikielny, C.W., J.L. Teem, and M. Rosbash. 1983. Evidence for the biochemical role of an internal sequence in yeast nuclear mRNA introns: implications for U1 RNA and metazoan mRNA splicing. Cell 34: 395-403.

Rogers, J. and R. Wall. 1980. A mechanism for RNA splicing. Proc. Natl. Acad. Sci. 77: 1877-1879.

Rothstein, R.J. 1983. One-step gene disruption in yeast. Methods Enzymol. 101: 202-211.

Sanger, F., S. Nicklen, and A.R. Coulson. 1977. DNA sequencing with chain-terminating inhibitors. Proc. Natl. Acad. Sci. 74: 5463-5467.

Siliciano, P.G., D.A. Brow, H. Roiha, and C. Guthrie. 1987a. An essential snRNA from $S$. cerevisiae has properties predicted for U4, including interaction with a U6-like snRNA. Cell 50: $585-592$.

Siliciano, P.G., M.H. Jones, and C. Guthrie. 1987b. Saccharomyces cerevisiae has a Ul-like small nuclear RNA with unexpected properties. Science 237: 1484-1487.

Teem, J.L., J. Abovich, N.F. Kaufer, W.F. Schwindinger, J.R. Warner, A. Levy, J. Woolford, R.J. Leer, M.M.C. van Raamsdonk-Duin, W.H. Mager, R.J. Planta, L. Schultz, J.D. Friesen, H. Fried, and M. Rosbash. 1984. A comparison of yeast ribosomal protein gene DNA sequences. Nucleic Acids Res. 12: 8295-8312.

Vijayraghavan, U., R. Parker, J. Tamm, Y. Iimura, J. Rossi, J. Abelson, and C. Guthrie. 1986. Mutations in conserved intron sequences affect multiple steps in the yeast splicing pathway, particularly assembly of the spliceosome. EMBO J. 5: $1683-1695$.

Weber, S. and M. Aebi. 1988. In vitro splicing of mRNA precursor: 5' cleavage site can be predicted from the interaction between the $5^{\prime}$ terminus of U1 snRNA. Nucleic Acids Res. 16: $471-486$

Wise, J.A., D. Tollervey, D. Maloney, H. Swerdlow, E.J. Dunn, and C. Guthrie. 1983. Yeast contains small nuclear RNAs encoded by single copy genes. Cell 35: 743-751.

Zhuang, Y., H. Leung, and A.M. Weiner. 1987. The natural 5' splice site of simian virus 40 large $\mathrm{T}$ antigen can be improved by increasing the base complementarity to U1 RNA. Mol. Cell. Biol. 7: 3018-3020.

Zhuang, Y. and A.M. Weiner. 1986. A compensatory base change in Ul snRNA suppresses a $5^{\prime}$ splice site mutation. Cell 46: 827-835. 


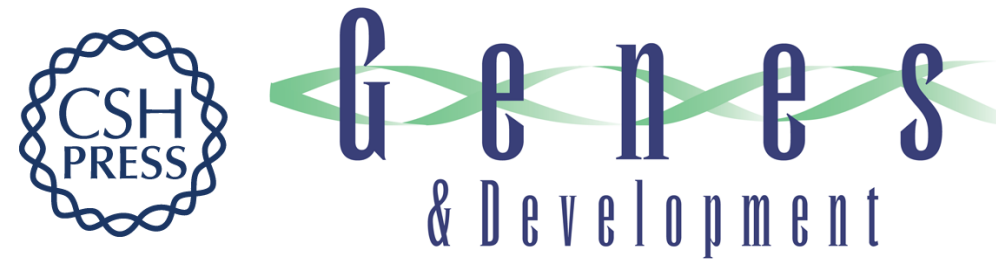

\section{5' splice site selection in yeast: genetic alterations in base-pairing with $U 1$ reveal additional requirements.}

P G Siliciano and C Guthrie

Genes Dev. 1988, 2:

Access the most recent version at doi:10.1101/gad.2.10.1258

References This article cites 39 articles, 11 of which can be accessed free at:

http://genesdev.cshlp.org/content/2/10/1258.full.html\#ref-list-1

License

Email Alerting

Service

Receive free email alerts when new articles cite this article - sign up in the box at the top right corner of the article or click here.

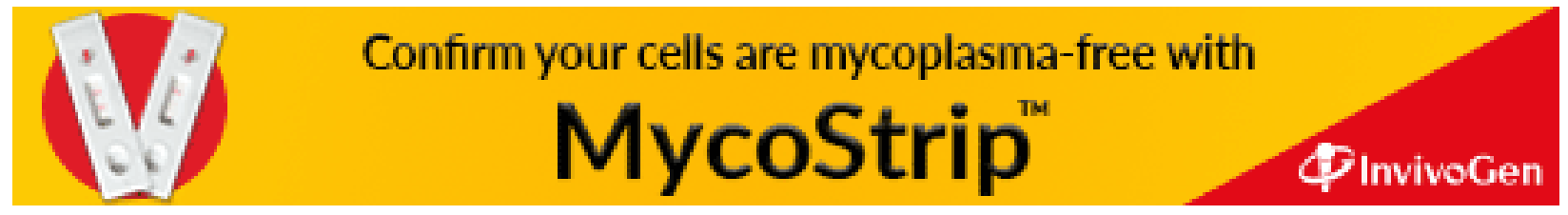

